\title{
Sekundäre Sectio: Was bringt die Vaginaldesinfektion?
}

La Rosa M. et al. Institutional protocols for vaginal preparation with antiseptic solution and surgical site infection rate in women undergoing cesarean delivery during labor. Obstet Gynecol 2018; 132: 371-376

\section{Zusammenfassung der Studie}

Mütter, die per Kaiserschnitt entbunden werden, haben im Vergleich zu Müttern mit Vaginalgeburt ein erhöhtes Risiko für eine Endometritis sowie Infektionen des Operationsgebiets. Besonders gefährdet sind diesbezüglich Frauen mit sekundärer Sectio caesarea nach Einsetzen der Wehentätigkeit. Können vaginale Desinfektionsmaßnahmen dieses Risiko senken? Dieser Frage sind US-Wissenschaftler nachgegangen.

Sie haben die Daten der „Cesarean Section Optimal Antibiotic Prophylaxis“-Studie einer Sekundäranalyse unterzogen. Im Rahmen dieser randomisierten, kontrollierten Multicenterstudie war untersucht worden, ob im Hochrisikokollektiv (Mütter mit Sectio caesarea nach Einsetzen der Wehentätigkeit, Gestationsalter $\geq 24$ Schwangerschaftswochen) durch die zusätzliche Gabe von Azithromycin das postoperative Infektionsrisiko gesenkt werden kann. 523 der insgesamt 2013 Studienteilnehmerinnen (26\%) wurden in Kliniken behandelt, die routinemäßig eine präoperative vaginale Desinfektion vornahmen. In 95\% der Fälle kam hierbei ein jodhaltiges Antiseptikum und in den übrigen Fällen Chlorhexidin zum Einsatz. Primärer Studienendpunkt war die Infektionsrate (oberflächliche/tiefe Wundinfektion, Organinfektion) innerhalb von 6 Wochen post partum. Die maternalen Outcomeparameter umfassten zusätzlich die Endometritis, postoperatives Fieber, die Länge des Klinikaufenthalts, die Wiederaufnahmerate, die Häufigkeit unerwarteter ärztlicher Konsultationen sowie Vorstellungen in der Notaufnahme. Die neonatalen Outcomeparameter umfassten Tod, Sepsis sowie positive Blut- und Liquorkulturen.

\section{Ergebnisse}

Die 523 vaginal antiseptisch vorbehandelten Studienteilnehmerinnen und die 1490 nicht vorbehandelten Kontrollen unterschieden sich hinsichtlich der Gabe prophylaktischer Antibiotika bzw. der Azithromycin-Randomisierung nicht. Bezüglich des kombinierten primären Studienendpunkts, dem Risiko für Infektionen im Operationsgebiet, fanden sich ebenfalls keine signifikanten Unterschiede zwischen den beiden Gruppen (10,5 vs. 8,5\%; Odds Ratio 1,27; $95 \%-K I$ 0,91-1,78). Die Nichtsignifikanz persistierte auch bei Berücksichtigung potenzieller Einflussvariablen. Mit Ausnahme der Dauer des Klinikaufenthalts die vaginal-antiseptisch vorbehandelten
Frauen blieben signifikant seltener länger als 5 Tage in der Klinik (1,7 vs. 5,2\%; $\mathrm{p}=$ $0,001)$ - ließen sich für keinen der übrigen maternalen Studienendpunkte signifikante Unterschiede zwischen der Antiseptika- und der Kontrollgruppe nachweisen. Bei den in den Kliniken mit routinemäßiger Vaginaldesinfektion geborenen Kindern wurde signifikant seltener der Verdacht auf eine Sepsis gestellt (5,4 vs. 14,5\%; Odds Ratio 0,33; 95\%-KI 0,22-0,50; $p<0,001$ ), die Rate bestätigter Sepsisfälle, die Mortalität sowie die Rate positiver Blut- und Liquorkulturen war dagegen in beiden Gruppen ähnlich.

FAZIT

Die Wissenschaftler schlussfolgern: Bei Müttern mit einer sekundären Sectio caesarea lässt sich offenbar durch eine Vaginaldesinfektion die Rate von Infektionen im Operationsgebiet nicht senken.

\section{Quelle}

Sekundäre Sectio: Was bringt die Vaginaldesinfektion?. Frauenheilkunde up2date 2018; 12(06): 495-496. doi:10.1055/a0808-0311 